\title{
OCT Angiographic changes in diabetic patients after cataract surgery
}

\author{
Radwa Sami Malik Ahmed, Hossam Eldin Mohamed Ahmed Khalil and Asmaa Mohamed Samir \\ Ophthalmology department, Faculty of Medicine, Beni-Suef University, Egypt
}

radwa.sami89@gmail.com;

\begin{abstract}
The goal of this prospective non-interventional clinical study was to evaluate the role of OCT-A parameters in determining early changes that occur in DM patients following cataract surgery to be aware about early microvascular changes occurring \& when to interfere to prevent complications of DR. The study was carried out on 30 eyes of cataractous diabetic patients without DR \& 30 eyes of cataractous non diabetic patients, both underwent phaco. Patients were selected according to inclusion \& exclusion criteria then complete ophthalmologic evaluation was done (history, examination and investigations). OCT-A was carried out for study groups before \&during follow up after one week, one month \&three months after surgery. The study showed that there was a significant increase in foveal \& parafoveal thickness after three months in comparison with preoperative values \& control group by $12 \pm 8.97$ foveal/ $11.40 \pm 3.99$ parafoveal(p-value $<0.001)$, also showed that there was a significant reduction in blood vessel density in SCP in 2 areas by 3.96 \pm 2.48 foveal $/ 6.46 \pm 2.04$ parafoveal ( $p$-value $<\mathbf{0 . 0 0 1}$ )after three months postoperative. It can be concluded that using OCT-A to evaluate VD in DM patients is efficient to detect early changes that occur before clinical manifestation.
\end{abstract}

Keywords : OCT Angiographi, cataract surgery, diabetic retinopathy, fovea, macula.

\begin{tabular}{|l|l|l|}
\hline Receive Date $: 14 / 1 / 2021$ & Accept Date: $14 / 2 / 2021$ & Publish Date : 1/4/2021 \\
\hline
\end{tabular}

\section{Introduction}

In 2000,the global prevalence of DM was estimated to be $2.8 \%$ and is thought to be $4.4 \%$ by 2030 . The total number of diabetic patients all over the world has risen from 171 million in 2000 to 366 million in 2030 (1). Diabetic retinopathy is one of the commonest causes of novel cases of blindness in adults between the ages of 20- 74 years. During the 1 st 2 decades of the disease, approximately, all patients with type 1 diabetes(T1DM) and more than $60 \%$ of patients with type 2 diabetes(T2DM)have retinopathy. In the Wisconsin Epidemiologic Study of Diabetic Retinopathy (WESDR), 3.6\% of younger-onset T1DM and $1.6 \%$ of olderonset T2DM were legally blind. In the youngeronset group, $86 \%$ of blindness resulted from diabetic retinopathy. In the older-onset group, in which other eye problems were common,1/3of cases of blindness were caused by diabetic 
retinopathy (2). In addition, cataract results at an earlier age and more frequent 5 times diabetic patients. Indeed, loss of vision has a noticeable effect on the working population $(3,4)$. Epidemiologic studies have determined that cataract is one of commonest causes of visual affection in older-onset DM patients and the rate of cataract surgery is subsequently elevated $(\mathbf{5 , 6 )}$. In fact,cataract surgeries help improving the outcome of visual acuity. However,not all patients have similar results, complications happen at various degrees such as retinopathy progression, neovascularizations (NVD or NVE), macular edema or decreased visual acuity. The progression of diabetic retinopathy, especially in studies that evaluated ECCE \& ICCE, might result from breakdown of the blood ocular barrier or increased inflammation in DM patients following extraction of cataract. The smaller size of incision along with shorter duration of surgery in phacoemulsification reduce inflammation and produce less breakdown of the blood ocular barrier. The absence of clinically significant influences in specific studies doesn't negate any undesirable effects from surgery. One day following quiet phaco and IOL implantation, the concentration of VEGF, hepatocyte growth factor,interleukin1 and pigment epithelium derived factor increases and need one month to be reduced to the preoperative level. These findings ensure changed concentrations of angiogenic and growth factors following cataract surgery that might enhance subclinical or even clinical aggravation of diabetic retinopathy and maculopathy (7). Changed concentrations of angiogenicfactors that occur following cataract surgery might cause worsening of maculopathy. After uneventful cataract surgery, OCT imaging has exhibited higher retinal thickness in diabetic eyes with no retinopathy in comparison with nondiabetics. Nevertheless, there was a trend toward more increased retinal thickness in diabetic that was evident up to three months following surgery $\mathbf{( 7 \& 8 )}$. In eyes with no clinically significant macular edema(CSME) at surgery, either due to the condition hadn't developed or was successfully managed, Early treatment diabetic retinopathy study (ETDRS) reveal number 25 exhibited that there was no statistically significant variation in the prevalence of macular edema prior to and 1 year following surgery (9). Also, another study documented that the incidence of new clinically detectable macular edema 1 year following surgery was nearly 56\%. However, within 6 months,spontaneous resolution without therapy occurred in about $50 \%$ of cases, and in approximately75\%by a year follow surgery (10). Other data revealed that macular edema usually occurs after cataract surgery;however, it might follow a benign course and in same lot of patients the development of CSME postoperatively may consider a progression in 
the normal course of the disease rather than a direct influence of surgery (11). So before the cataract surgery,it is better for the patients to have good glycemic control and to be sure of absent ocular or periocular infection. Additionally,patients should be subjected to full ophthalmologic examination that include evaluation of visual acuity (VA), relative afferent pupillary defect (RAPD), best corrected visual acuity (BCVA), slitlamp biomicroscopy, gonioscopy (with special attention to novel vessels), tonometry. Dilated funduscopy is a must. Supplementary diagnostic assessment as fluorescein angiography,optical coherence tomography (OCT),OCTangiography and B-scanultrasonography might help selected cases (12). It is preferred to use OCT-angiography (OCT-A) in these cases as it shows more details in the superficial as well as in the deep retinal layer than FA (13). Several studies have shown that OCT-A is useful in the diagnosis of choroidal neovascularization $(\mathrm{CNV})$ age-related macular degeneration(AMD), (14) retinal vein occlusion (RVO), (13) altered retinal vessels (15), nonexudativeAMD(16)and melanocytic tumors (17). But in contrast to FA, OCT-A can't determine pooling staining or leakage (14). As OCT-A is a technique that depends on motion detection, structures with very slow motion(flow) will be hardly visible. Furthermore, changes in the retinal thickness may result in visual reposition of the vessels
(18). So It is considered a reliable procedure that can be used in the pre-operative examination \& post-operative follow-up of patients in order to detect any alterations in retinal vasculature that allow for early intervention \& good results for patient (18). This study aimed to evaluate the role of OCTangiography in detecting changes that occur in DM patients following cataract surgery in comparison with non-diabetic patients, also early diabetic retinopathy features to be aware about early microvascular changes that occur in diabetics \& when to interfere to prevent DR complications.

\section{Patients and Methods}

This is a prospective, non-randomized, comparative, non-interventional clinical case study was done in Ophthalmology department of Beni-Seuf University Hospital on 30 eyes of cataractou sdiabetics without DR \& 30 eyes of cataractous non diabetic patients. Both groups undergo cataract surgery (phacoemulsification). OCT-Angiography was done to study groups before \&during follow up after one week, one month \&three months after surgery. All patients were fully informed about the procedure \&had signed an informed consent. The protocol of this research was approved by research ethics committee of Faculty of Medicine of BSU.

\subsection{Inclusion criteria:}

1. Patients with T2DM without DR manifested clinically or by OCT \& had cataract.

2. Age group is from $30-60$ 


\section{Exclusion criteria:}

1. Patients who refused to enroll in the study.

2. Diabetic patients with retinopathy.

3. Patients with media opacities that will disturb

4. OCT-A performance.

5. Patients with history of any retinal vascular disease.

6. Patients with history of eye trauma.

7. Patients who did extra capsular cataract extraction (ECCE).

8. Patients with complicated procedure.

9. Years old for both diabetic \&non diabetic patients.

\subsection{All patients were subjected to:}

Enrolled patients were evaluated by complete ophthalmologic evaluation as following:

History: Personal data (name, age, sex, residency, telephone number and occupation), data related to: medical condition \& current medications used and data related to exclusion criteria.

\section{Examination:}

○ Visual acuity assessment: unaided \& aided using Landolt Cchart

○ External examination: lids, lashes, lacrimal apparatus and orbit.

$\bigcirc$ Examination of ocular alignment and motility

○ Assessment of papillary function.

○ Measurement: using Goldmann aplanation tonometer.

○ Slit-Lamp biomicroscopic examination.
O Optic nerve and vitreous.

Dilated examination: of the lens, macula by Volk's non-contact double aspheric biconvex lens (power: +90D), peripheral retina (by Indirect ophthalmoscopy),

Optical coherence tomography angiography (OCT-A)

[AngioVuesoftware (software version: 2016.0.0.52) of the RTVueXR Avantispectraldomain OCT (SD-OCT) (Optovue, Inc, Fremont, CA)] was carried out for patients to evaluate the macular thickness \& vasculature of macular area before phacoemulsification then done one week, one month \& three months of follow uptime.

\section{Procedures}

Patients from outpatient clinic, who were diagnosed diabetic with no clinically manifest DR \& had cataract, after full clinical examination OCT-A was carried out before phaco and similar was done for normal control cases. All patients followed after one week, one month \& three months by clinical examination \& OCT-A imaging in order to detect any changes occured following phaco either clinically significant or by investigation. OCTA imaging was done by AngioVue software (software version: 2016.0.0.52) of the RTVueXRA vantispectral-domain OCT(SD-OCT) after full dilatation of pupil with scan size $6 \times 6 \mathrm{~mm}$ to document any changes resulted between before\&after phaco in central macular thickness, FAZ changes (can't be measured by used software) \& microvasculature density of macular area in superficial \&deep layers (deep 
layer vascular density can't be assessed by used software). Data collected continuously after every follow up visit.

\section{Statistical methodology}

Data were manipulated, edited, and analyzed by IBM SPSS ${ }^{\circ} \quad$ (version 22.0) Software. Descriptive statistics for continuous variables were presented as mean, standard deviation (SD), minimum, and maximum values. Categorical variables were described as frequency $\& \%$. The differences were tested for statistical significance using paired t-test.

The comparison between groups concerning qualitative data was carried out using chisquare test. Differences between postoperative measurements were presented as mean difference, $95 \%$ confidence interval \& the margin of error accepted was set to $5 \%$.

\section{Results}

A total of 60 eyes fulfilling the inclusion criteria for this study were included and divided into two groups: Diabetic group: group of 30 eyes with age ranged from $40-62$ with mean \pm SD (52.76 \pm 6.04$)$. They were 12 male $(40 \%) \& 18$ female (60\%). Control group (non-diabetic): group of 30 eyes with age ranged from 35-63 withmean $\pm \mathrm{SD}(52.40 \pm 7.77)$. They were 20 male $(66.7 \%) \& 10$ female $(33.3 \%)$.

Table (1): Demographic data of studied groups

\begin{tabular}{|l|c|c|c|}
\hline & $\begin{array}{c}\text { Non diabetic } \\
\text { group N=30 }\end{array}$ & $\begin{array}{c}\text { Diabetic group } \\
\mathbf{N = 3 0}\end{array}$ & P-value \\
\hline Age in years & $\begin{array}{l}5.40 \pm 7.77 \\
\text { Mean } \pm \text { SD }\end{array}$ & $\begin{array}{c}52.76 \pm 6.04 \\
40-62\end{array}$ & 0.839 \\
Range & $35-63$ & & \\
\hline Sex: $\mathrm{n}(\%)$ & & $12(40 \%)$ & 0.069 \\
Males & $20(66.7 \%)$ & $18(60 \%)$ & \\
Females & $10(33.3 \%)$ & \\
\hline
\end{tabular}

Table (1) shows that there was

insignificant difference between diabetic \& nondiabetic groups regarding age $\&$ gender.

Table (2): Comparison between studied groups regarding fovea thickness in micron

\begin{tabular}{|c|c|c|c|}
\hline \multirow[t]{2}{*}{ Fovea thickness in micron } & $\begin{array}{l}\text { Nondiabetic group } \\
\mathrm{N}=30\end{array}$ & $\begin{array}{c}\text { Diabetic group } \\
\qquad \mathrm{N}=30\end{array}$ & \multirow{2}{*}{$\begin{array}{c}\text { P-value (2 } \\
\text { groups) }\end{array}$} \\
\hline & Mean \pm SD & Mean \pm SD & \\
\hline Before & $235.06 \pm 8.92$ & $248.16 \pm 11.93$ & $<0.001 * * *$ \\
\hline After $1 \mathrm{w}$ & $236.13 \pm 8.61$ & $250.36 \pm 11.59$ & $<0.001 * * *$ \\
\hline After $1 \mathrm{~m}$ & $236.80 \pm 9.58$ & $255.10 \pm 12.02$ & $<0.001 * * *$ \\
\hline After $3 \mathrm{~m}$ & $236.06 \pm 8.86$ & $260.16 \pm 14.88$ & $<0.001 * * *$ \\
\hline \multicolumn{4}{|l|}{ p-value before $\&$ after } \\
\hline Before \& 1w & $0.047 *$ & $<0.001 * * *$ & \\
\hline Before \& 1m & $<0.001 * * *$ & $<0.001 * * *$ & \\
\hline Before \& 3m & $0.004 * *$ & $<0.001 * * *$ & \\
\hline $1 \mathrm{w} \& 1 \mathrm{~m}$ & 0.277 & $<0.001 * * *$ & \\
\hline $1 \mathrm{w} \& 3 \mathrm{~m}$ & 0.890 & $<0.001 * * *$ & \\
\hline $1 \mathrm{~m} \& 3 \mathrm{~m}$ & $0.015^{*}$ & $<0.001 * * *$ & \\
\hline
\end{tabular}

Paired t-test $*$ significant. $\quad-\quad$ - $*$ moderately significant. $\quad-\quad * * *$ highly significant. 
Table (2) shows that there was high statistically significant increase in foveal thickness between diabetic\&control groups before \& after surgery. There was also a high significant increase in diabetic group before, after 1week, after 1month \&after 3months of follow up post-surgery, unlike the control group before $\&$ after surgery.

Table (3): Comparison between studied groups regarding fovea thickness (in micron) increase

\begin{tabular}{|l|c|c|c|}
\hline \multirow{2}{*}{ Fovea thickness (in micron) increase } & $\begin{array}{c}\text { Non diabetic group } \\
\mathbf{N}=\mathbf{3 0}\end{array}$ & Diabetic group $\mathbf{N}=\mathbf{3 0}$ & \multirow{2}{*}{ P-value } \\
\cline { 2 - 3 } & Mean \pm SD & $\begin{array}{c}\text { Mean } \pm \text { SD } \\
\text { (median) }\end{array}$ & \\
\hline After $1 \mathrm{w}$ & $1.06 \pm 2.81(2)$ & $2.20 \pm 2.78(2)$ & 0.122 \\
\hline After $1 \mathrm{~m}$ & $1.73 \pm 1.72(2)$ & $6.93 \pm 6.44(5)$ & $<0.001^{* * *}$ \\
\hline After $3 \mathrm{~m}$ & $1 \pm 1.74(1)$ & $12 \pm 8.97(10)$ & $<0.001^{* * *}$ \\
\hline
\end{tabular}

Table (3) shows that there was high statistically significant increase in thickness especially post surgery long term follow up in comparison with the preoperative in DM group mainly.

Table (4): Comparison between studied groups regarding fovea Blood vessel density \%

\begin{tabular}{|l|c|c|c|}
\hline \multirow{2}{*}{$\begin{array}{c}\text { Fovea Blood vessel density } \\
\text { \% }\end{array}$} & $\begin{array}{c}\text { Non diabetic group } \\
\mathrm{N}=30\end{array}$ & $\begin{array}{c}\text { Diabetic group } \\
\mathrm{N}=30\end{array}$ & $\begin{array}{c}\text { P-value }(2 \\
\text { groups })\end{array}$ \\
\cline { 2 - 3 } & $\mathrm{Mean} \pm \mathrm{SD}$ & $\mathrm{Mean} \pm \mathrm{SD}$ & $0.049^{*}$ \\
\hline Before & $33.06 \pm 6.11$ & $30.23 \pm 4.71$ & $0.015^{*}$ \\
\hline After 1 w & $31.86 \pm 6.12$ & $28.33 \pm 4.68$ & 0.084 \\
\hline After 1 m & $31.13 \pm 6.05$ & $28.53 \pm 5.37$ & $0.001^{* *}$ \\
\hline After 3 m & $31.40 \pm 5.82$ & $26.26 \pm 5.23$ & \\
\hline p-value before \& after & \multicolumn{3}{|c|}{} \\
\hline Before \& 1w & $<0.001^{* * *}$ & $<0.001^{* * * *}$ & \\
\hline Before \& 1m & $<0.001^{* * *}$ & $0.006^{* *}$ & \\
\hline Before \& 3m & $<0.001^{* * *}$ & $<0.001^{* * *}$ & \\
\hline $1 \mathrm{w} \& 1 \mathrm{~m}$ & $0.001^{* *}$ & 0.727 & \\
\hline $1 \mathrm{w} \& 3 \mathrm{~m}$ & 0.075 & $0.001^{* *}$ & \\
\hline $1 \mathrm{~m} \& 3 \mathrm{~m}$ & 0.442 & $<0.001^{* * *}$ & \\
\hline
\end{tabular}

Table (4) shows that there was statistically significant decrease in foveal blood vessels density between diabetic\&control groups before \& after surgery. There was also high significant decrease in diabetic group before,after 1week\&after 3monthsof follow up mainly post-surgery in control group but in DM group the decrease in density was progressive unlike control group which stopped after 1month.

Table (5): Comparison between studied groups regarding fovea blood vessel density \% absolute reduction

\begin{tabular}{|l|c|c|c|}
\hline \multirow{2}{*}{$\begin{array}{c}\text { Fovea blood vessel density } \\
\% \text { absolute reduction }\end{array}$} & $\begin{array}{c}\text { Non diabetic group } \\
\mathrm{N}=30\end{array}$ & Diabetic group N=30 & \multirow{2}{*}{ P-value } \\
\cline { 2 - 3 } & Mean \pm SD & Mean \pm SD & $0.022^{*}$ \\
\hline After $1 \mathrm{w}$ & $1.20 \pm 0.99(1)$ & $1.90 \pm 1.29(2)$ & 0.698 \\
\hline After $1 \mathrm{~m}$ & $1.93 \pm 1.01(2)$ & $1.70 \pm 3.12(2)$ & $<0.001^{* * *}$ \\
\hline After $3 \mathrm{~m}$ & $1.66 \pm 1.60(2)$ & $3.96 \pm 2.48(4)$ & \\
\hline
\end{tabular}

Table (5) shows that there was high statistically significant decrease in vessel density particularly post-surgery long term follow up in comparison with preoperative in DM group mainly. 
Table (6): Comparison between studied groups regarding parafoveal thickness in micron

\begin{tabular}{|l|c|c|c|}
\hline \multirow{2}{*}{$\begin{array}{c}\text { Parafoveal thickness in } \\
\text { micron }\end{array}$} & $\begin{array}{c}\text { Non diabetic group } \\
\mathrm{N}=30\end{array}$ & $\begin{array}{c}\text { Diabetic group } \\
\mathrm{N}=30\end{array}$ & \multirow{2}{*}{$\begin{array}{c}\text { P-value }(2 \\
\text { groups })\end{array}$} \\
\cline { 2 - 3 } & $\mathrm{Mean} \pm \mathrm{SD}$ & Mean \pm SD & 0.561 \\
\hline Before & $301.93 \pm 8.56$ & $299.80 \pm 18.05$ & 0.817 \\
\hline After 1 w & $302.80 \pm 8.47$ & $301.93 \pm 18.58$ & 0.656 \\
\hline After 1 m & $305.33 \pm 9.18$ & $307.03 \pm 18.65$ & 0.145 \\
\hline After 3 m & $305.26 \pm 9.42$ & $311.20 \pm 19.90$ & \\
\hline p-value before \& after & \multicolumn{3}{|c|}{} \\
\hline Before \& 1w & $0.001^{* *}$ & $<0.001^{* * *}$ & \\
\hline Before \& 1m & $<0.001^{* * *}$ & $<0.001^{* * *}$ & \\
\hline Before \& 3m & $<0.001^{* * *}$ & $<0.001^{* * *}$ & \\
\hline $1 \mathrm{w} \& 1 \mathrm{~m}$ & $<0.001^{* * *}$ & $<0.001^{* * *}$ & \\
\hline $1 \mathrm{w} \& 3 \mathrm{~m}$ & $<0.001^{* * *}$ & $<0.001^{* * *}$ & \\
\hline $1 \mathrm{~m} \& 3 \mathrm{~m}$ & 0.860 & $<0.001^{* * *}$ & \\
\hline
\end{tabular}

Table (6) shows that there was statistically insignificant increase in parafoveal thickness between diabetic\&control groups before $\&$ after surgery. There was also a high significant increase in DM group before,after 1week, after 1month \&after 3months of follow up post surgery as well as control group except after 3 months.

Table (7): Comparison between studied groups regarding parafoveal thickness absolute increase (in micron)

\begin{tabular}{|l|c|c|c|}
\hline \multirow{2}{*}{$\begin{array}{c}\text { Parafoveal thickness } \\
\text { absolute increase (in } \\
\text { micron) }\end{array}$} & $\begin{array}{c}\text { Non diabetic group } \\
\mathbf{N}=\mathbf{3 0}\end{array}$ & $\begin{array}{c}\text { Diabetic } \\
\text { group N=30 }\end{array}$ & \multirow{2}{*}{ P-value } \\
\cline { 2 - 3 } & $\mathbf{M e a n} \pm$ SD & Mean \pm SD & \\
\hline After 1 w & $0.86 \pm 1.22$ & $2.13 \pm 2.01$ & 0.005 \\
\hline After 1 m & $3.40 \pm 2.19$ & $7.23 \pm 3.23$ & $<0.001^{* * *}$ \\
\hline After 3 m & $3.33 \pm 2.45$ & $11.40 \pm 3.99$ & $<0.001^{* * *}$ \\
\hline
\end{tabular}

Table (7) shows that there was high statistically significant increase in thickness especially post surgery long term follow up in comparison with preoperative in DM group mainly.

Table (8): Comparison between studied groups regarding parafoveal Blood vessel density \%

\begin{tabular}{|l|c|c|c|}
\hline \multirow{2}{*}{$\begin{array}{c}\text { Parafoveal Blood vessel } \\
\text { density \% }\end{array}$} & $\begin{array}{c}\text { Non diabetic group } \\
\mathbf{N = 3 0}\end{array}$ & $\begin{array}{c}\text { Diabetic group } \\
\text { N=30 }\end{array}$ & \multirow{2}{*}{$\begin{array}{c}\text { P-value (2 } \\
\text { groups) }\end{array}$} \\
\cline { 2 - 3 } & Mean \pm SD & Mean \pm SD & 0.696 \\
\hline Before & $48.93 \pm 4.10$ & $49.30 \pm 3.03$ & 0.867 \\
\hline After 1 w & $47.73 \pm 4.33$ & $47.90 \pm 3.27$ & 0.130 \\
\hline After 1 m & $46.93 \pm 4.35$ & $45.36 \pm 3.48$ & $<0.001^{* * *}$ \\
\hline After 3 m & $46.93 \pm 3.62$ & $42.83 \pm 3.46$ & \\
\hline p-value before \& after & \multicolumn{3}{|c|}{} \\
\hline Before \& 1w & $<0.001^{* * *}$ & $<0.001^{* * *}$ & \\
\hline Before \& 1m & $<0.001^{* * *}$ & $<0.001^{* * *}$ & \\
\hline Before \& 3m & $<0.001^{* * *}$ & $<0.001^{* * *}$ & \\
\hline $1 \mathrm{w} \& 1 \mathrm{~m}$ & $0.016^{*}$ & $<0.001^{* * *}$ & \\
\hline $1 \mathrm{w} \& 3 \mathrm{~m}$ & $0.004^{* * *}$ & $<0.001^{* * *}$ & \\
\hline $1 \mathrm{~m} \& 3 \mathrm{~m}$ & 1.000 & $<0.001^{* * *}$ & \\
\hline
\end{tabular}

Table (8) shows that there was statistically insignificant decrease in parafoveal blood vessels density between diabetic \& control groups before $\&$ after surgery except after 3 months of follow up there was high significant decrease. There was also high significant decrease in DM group before, after 
1 week \& after 3months of follow up particularly post surgery also in control group but in DM group the decrease in density was progressive unlike control group which stopped after 1month.

Table (9): Comparison between studied groups regarding parafoveal blood vessel density \% absolute reduction

\begin{tabular}{|l|c|c|c|}
\hline $\begin{array}{c}\text { Parafoveal blood vessel } \\
\text { density } \begin{array}{c}\text { \% absolute } \\
\text { reduction }\end{array}\end{array}$ & $\begin{array}{c}\text { Non diabetic group } \\
\mathbf{N}=\mathbf{3 0}\end{array}$ & Diabetic group $\mathbf{N = 3 0}$ & \multirow{2}{*}{ P-value } \\
\cline { 2 - 3 } & Mean $\mathbf{\pm S D}$ & Mean $\mathbf{\pm S D}$ & 0.541 \\
\hline After $1 \mathrm{w}$ & $1.20 \pm 1.12$ & $1.40 \pm 1.37$ & $<0.001 * * *$ \\
\hline After $1 \mathrm{~m}$ & $2 \pm 1.61$ & $3.93 \pm 2.28$ & $<0.001 * * *$ \\
\hline After $3 \mathrm{~m}$ & $2 \pm 1.23$ & $6.46 \pm 2.04$ & $<$ \\
\hline
\end{tabular}

Table (9) shows that there was high statistically significant decrease in vessel density especially post surgery long term follow up in comparison with preoperative mainlyin DM group.

\section{Discussion}

The aim of the study was to investigate the effect of uncomplicated standard phaco with IOL implantation on the central retina in DM patients without any changes preoperatively and non DM subjects in the early \& late postoperative period of three months by OCT$\mathrm{A}$, and it revealed that surgery affects diabetic retina more than control retina.

The commonest change that known to happen following phaco is increase the thickness of central macular area in DM patients in comparison with non-diabetic group the increase in macular thickness (in foveal \& parafoveal areas) was highly significant by Mean \pm SD 248.16 $\pm \mathbf{1 1 . 9 3}$ compared to 235.06 \pm 8.92 (p-value $<\mathbf{0 . 0 0 1 )}$ of non-diabetic preoperatively up to $\mathbf{2 6 0 . 1 6} \pm \mathbf{1 4 . 8 8}$ compared to $\mathbf{2 3 6 . 0 6} \pm \mathbf{8 . 8 6}$ (p-value $<\mathbf{0 . 0 0 1}$ ) of non-diabetic after postoperative follow up for three months.

These results, in general, in agreement with other studies made to prove the effect of cataract surgery on macular thickness of DM retina as the study results Degenring et al. ${ }^{(19)}$ that concluded that the foveal thickness FT increased from $183 \pm 27 \mu \mathrm{m}$ preoperatively to $191 \pm 37 \mu \mathrm{m}$ four weeks following surgery ( $p=0.001)$, with DM patients exhibiting a tendency toward a more pronounced increase in minimal retinal thickness than non-diabetic subjects $(p=0.058)$. One day $\&$ one week after procedure, FT measurements weren't significantly different from preoperative results. Foveal volume showed a significant increase at one week \& four weeks following surgery $(p<0.001)$, independent of the presence of diabetes $(p=0.565)$. The proportion of patients exhibiting subclinical macular swelling was about $1 / 5$ in the non-diabetic group and $1 / 3$ in the diabetic group. Mean duration of surgery was $11.5 \pm 6.6 \mathrm{~min}$.

However, there was a slight difference from results of Katsimpris et al. (20) study that reported that the preoperative central foveal thickness CFT showed no significant difference between the 2groups (normal: $205 \pm 18 \mu \mathrm{m}$ vs. diabetics: $202 \pm 23 \mu \mathrm{m}, \mathrm{p}>0.1)$. Postoperative 
CFT in DM patients at all time-points of the follow-up period was significantly increased when compared to controls $\left(1^{\text {st }}\right.$ month, normals: $215 \pm 28 \mu \mathrm{m}$ vs. diabetics: $262 \pm 33 \mu \mathrm{m}, \mathrm{p}<0.05$; $3^{\text {rd }}$ third month, normal: $211 \pm 19 \mu \mathrm{m}$ vs. diabetics: $250 \pm 27 \mu \mathrm{m}, \mathrm{p}<0.05 ; 6^{\text {th }}$ month, normal: $208 \pm 12 \mu \mathrm{m}$ vs. diabetics: $266 \pm 13 \mu \mathrm{m}$, $\mathrm{p}<0.05$; and $12^{\text {th }}$ month, normal: $209 \pm 13 \mu \mathrm{m}$ vs. diabetics: $280 \pm 11 \mu \mathrm{m}, \mathrm{p}<0.05)$. The incidence of cystoid macular edema (CME)was $4.0 \%$ and $28.6 \%$ for the control group and the DM group, respectively, at the end of the follow-up period $(\mathrm{p}<0.05)$.

Other changes detected by OCT-A that indicated subclinical changes occurring in retinal microvasculature which was still progressive up to three months postoperative as decrease in blood vessel density VD in superficial capillary plexus SCP in foveal \& parafoveal areas as following:

- In foveal area of DM group blood VD in SCP showed reduction between preoperative Mean \pm SD $30.23 \pm 4.71 \&$ postoperative values by Mean \pm SD28.33 \pm 4.68 after one week, $28.53 \pm 5.37$ after one month, $26.26 \pm 5.23$ after 3 months with reduction Mean \pm SD 3.96 $\pm 2.48(4)$ after 3 months and by highly significant difference between pre\&three months postoperative follow up period ( $\mathrm{p}$-value $<0.001$ ).

- On the other hand non-diabetic group also showed reduction between pre \& post operative values but with mild degrees by Mean \pm SD $1.66 \pm 1.60$ (2) after 3 months follow up.

- In parafoveal area blood VD in SCP showed reduction preoperatively Mean \pm SD 49.30 \pm 3.03 from postoperatively Mean \pm SD $47.90 \pm 3.27$ after one week, $45.36 \pm 3.48$ after one month, $42.83 \pm 3.46$ after three months with high significant difference in values before \& after 3 months follow up by Mean \pm SD $11.40 \pm 3.99$ (p-value <0.001).

- Unlikely in non-diabetic group reduction in blood VD in SCP is less than in diabetic group. Between pre\&post operative after three months follow up values difference by Mean \pm SD 3.33 \pm 2.45 .

These results are in agreement with studies made to detect early DM changes by OCT-A that occur in diabetics with no diabetic retinopathy NDR indicating progression to DR (but not related to cataract surgery or its effect on diabetic retina).

In a study made by Galina et al. (21) the results showed that in superficial\&deep retina, vessel densities in $\operatorname{NDR}(44.35 \% \pm 13.31 \%$ and $31.03 \% \pm 16.33 \%$ ) were decreased in comparison with control subjects(51.39\% \pm $13.05 \%, P=0.04$; and $41.53 \% \pm 14.08 \%, P<$ $0.01) \&$ superficial vessel density significantly correlated with full retinal thickness and volume in parafovea $(r=0.43, P=0.01 ; r=0.43, P=$ 0.01 ) and with outer retinal volume in parafovea 
( $r=0.35, P<0.05$ )of healthy subjects. This indicates that superficial \& deep retinal vessel density in parafovea of DM patients without diabetic retinopathy are both decreased in comparison with healthy subjects. The associations between vessel density with retinal tissue thickness and with subject's clinical characteristics vary between healthy subjects \& patients with NDR".

Another study made by Alice Y. Kim, et al (22) To quantify changes in retinal microvasculature in diabetic retinopathy (DR) showed" decreasing capillary density (VD),branching complexity (fractal diameter FD), and increasing average vascular caliber \{vessel diameter index (VDI) $\}$ were associated with aggravation of DR\&changes in capillary density and morphology were significantly correlated with diabetic macular edema".

There is a study made by Eladawi et al. (23) showed "OCT-A is a useful instrument for detection of the early changes that occur in DR before being manifested clinically.Changes on VD, FAZ \& blood vessels caliber are also important indicators for DR progression in patients follow up". A Prospective study made by Ishibazawa et al. (24) to evaluate imaging technology on 47eyes with DR scanned by highspeed 840-nm-wavelength spectral-domain optical coherence tomography instrument (RTVue XR Avanti; Optovue, Inc, Fremont, California, USA). Blood flow was detected using the split-spectrum amplitude-decorrelation angiography algorithm. Fluorescein angiography (FA) images were done to all eyes to visualize microaneurysms, retinal nonperfused areas, and neovascularization and compared with that of the OCT angiograms. The results were: In 42 eyes, microaneurysms detected by FA near the macula appeared as focally dilated saccular or fusiform capillaries on OCT-A of the superficial and/or deep capillary plexus. Retinal nonperfused areas seen by FA appeared as lesions with no or sparse capillaries on OCT-A. Area measurement of retinal nonperfusion near the macula in 7 eyes revealed a difference between the extent of nonperfused areas in superficial and deep plexuses. In 4 eyes, the vascular structures of neovascularization at the optic disc were clearly visualized on OCT-A. At the end of the study they recommended use of OCT-A as it clearly visualize microaneurysms and retinal nonperfused areas and enables closer observation of each layer of the retinal capillaries. Quantitative information on new vessels can also be obtained. OCT-A may be clinically useful to evaluate the microvascular status and therapeutic effect of treatments for DR.

All previously mentioned studies discussing the early DR changes that can be detected by OCTA \& not manifested clinically making OCT-A one of the important tools in DM routine follow up; but regarding post cataract follow up, 
studies discussing this point are still unavailable.

By overall view to our results we found that OCT-A might be helpful in detection of the early diabetic changes, thus reducing related complications as it detect patients how need further investigations \&close follow up.

\section{Conclusion}

From the results of our study, OCT-A is helpful in diagnosis \& follow up of DM patients with early DR. It provides detailed imaging \&data of retinal layers microvascular changes that can't be detected by other investigation tools. Phaco affects the progression of DR on the long run indicating proper follow up for DM patients after surgery. CMT increases significantly in diabetics postoperative while VD in SCP decreases significantly when compared to preoperative $\&$ non-diabetic population. Role of OCT-A in the detection of the effect of cataract surgery needs more studies to be evaluated.

\section{References}

1. Wild S, Roglic G, Green A, Sicree R, King H. Global prevalence of diabetes: estimates for the year 2000 and projections for 2030 . Diabetes Care. 2004; 27(5):1047-53.

2. Fong DS, Aiello L, Gardner TW, King GL, Blankenship G, Cavallerano JD, et al. Retinopathy in Diabetes. Diabetes Care. 2004; 27(suppl 1):s84-7.
3. Klein BE, Klein R, Moss SE. Incidence of cataract surgery in the Wisconsin Epidemiologic Study of Diabetic Retinopathy. Am J Ophthalmol. 1995; 119(3):295-300.

4. Klein BE, Klein R, Wang Q, Moss SE. Older-onset diabetes and lens opacities. The Beaver Dam Eye Study. Ophthalmic Epidemiol. 1995; 2(1):49-55.

5. Klein BE, Klein R, Moss SE. Prevalence of cataracts in a population-based study of persons with diabetes mellitus. Ophthalmology. 1985; 92(9):1191-6.

6. Klein R, Klein BEK, Moss SE. Visual Impairment in Diabetes. Ophthalmology. 1984; 91(1):1-9.

7. Patel JI, Hykin PG, Cree IA. Diabetic cataract removal: postoperative progression of maculopathy growth factor and clinical analysis. Br J Ophthalmol. 2006; 90(6):697701.

8. Jurecka T, Bátková Z, Ventruba J, Synek S. [Macular edema after cataract surgery in diabetic patients without retinopathy]. Cesk Slov Oftalmol. 2007; 63(4):274-84.

9. Chew EY, Benson WE, Remaley NA, Lindley AA, Burton TC, Csaky K, et al. Results after lens extraction in patients with diabetic retinopathy: early treatment diabetic retinopathy study report number 25 . Arch Ophthalmol. 1999; 117(12):1600-6.

10. Dowler JG, Sehmi KS, Hykin PG, Hamilton AM. The natural history of macular edema after cataract surgery in diabetes. Ophthalmology. 1999; 106(4):663-8. 
11. Squirrell D, Bhola R, Bush J, Winder S, Talbot JF. A prospective, case controlled study of the natural history of diabetic retinopathy and maculopathy after uncomplicated phacoemulsification cataract surgery in patients with type 2 diabetes. $\mathrm{Br} \mathbf{J}$ Ophthalmol. 2002; 86(5):565-71.

12. Minckler D, Astorino A, Hamilton AMP. Cataract surgery in patients with diabetes. Ophthalmology. 1998; 105(6):949-50.

13. Mastropasqua R, Di Antonio L, Di Staso S, Agnifili L, Di Gregorio A, Ciancaglini M, et al. Optical Coherence Tomography Angiography in Retinal Vascular Diseases and Choroidal Neovascularization [Internet]. Journal of Ophthalmology. 2015

14. Marduel R. Angio OCT, Dye Less Angiography, A New Approach of Age Related Macular Degeneration (ARMD). Advances in Ophthalmology \& Visual System. 2015; 2.

15. Sharma P, Sridhar J, Rayess N, Maguire JI. Optical coherence tomography angiography (OCT-A) of type 2 retinal arteriovenous malformation. Can J Ophthalmol. 2015; 50(5):e93-96.

16. Schwartz DM, Fingler J, Kim DY, Zawadzki RJ, Morse LS, Park SS, et al. Phase-variance optical coherence tomography: a technique for noninvasive angiography. Ophthalmology. 2014; 121(1):180-7.

17. Maloca P, Gyger C, Hasler PW. A pilot study to image the vascular network of small melanocytic choroidal tumors with speckle noise-free 1050-nm swept source optical coherence tomography (OCT choroidal angiography). Graefes Arch Clin Exp Ophthalmol. 2016; 254(6):1201-10.

18. Zeimer M, Gutfleisch M, Heimes B, Spital G, Lommatzsch A, Pauleikhoff D. Association between Changes in Macular Vasculature in Optical Coherence Tomography- and Fluorescein- Angiography and Distribution of Macular Pigment in Type 2 Idiopathic Macular Telangiectasia. Retina. 2015; 35(11): 2307-2316.

19. Degenring RF, Vey S, Kamppeter B, Budde WM, Jonas JB, Sauder G. Effect of uncomplicated phacoemulsification on the central retina in diabetic and non-diabetic subjects. Graefes Arch ClinExpOphthalmol. 2007; 245(1):18-23.

20. Katsimpris JM, Petropoulos IK, Zoukas G, Patokos T, Brinkmann CK, Theoulakis PE. Central foveal thickness before and after cataract surgery in normal and in diabetic patients without retinopathy. Klin Monbl Augenheilkd. 2012; 229(4):331-7.

21. Dimitrova G, Chihara E, Takahashi H, Amano H, Okazaki K. Quantitative Retinal Optical Coherence Tomography Angiography in Patients With Diabetes Without Diabetic Retinopathy. Invest Ophthalmol Vis Sci. 2017; 58(1):190-6.

22. Kim AY, Chu Z, Shahidzadeh A, Wang RK, Puliafito CA, Kashani AH. Quantifying Microvascular Density and Morphology in Diabetic Retinopathy Using SpectralDomain Optical Coherence Tomography 
Angiography. Invest Ophthalmol Vis Sci. 2016; 57(9):OCT362-370.

23. Eladawi N, Elmogy M, Khalifa F, Ghazal M, Ghazi N, Aboelfetouh A, et al. Early diabetic retinopathy diagnosis based on local retinal blood vessel analysis in optical coherence tomography angiography
(OCTA) images. Med Phys. 2018; 45(10):4582-99.

24. Ishibazawa A, Nagaoka T, Takahashi A, Omae T, Tani T, Sogawa K, et al. Optical Coherence Tomography Angiography in Diabetic Retinopathy: A Prospective Pilot Study. Am J Ophthalmol. 2015; 160(1):3544.e1. 